\title{
Adenoma pleomórfico en paladar
}

\author{
Casos Clínicos
}

\author{
Pleomorfhic Adenoma in palate
}

\section{Resumen}

El adenoma pleomórfico, también conocido como tumor mixto benigno, es la neoplasia más común tanto en glándulas salivales mayores como en menores. Suele presentarse como una lesión firme de crecimiento lento y asintomático. En glándulas salivares menores, el sitio preferencial está en el paladar, seguido del labio superior y mucosa yugal. Su diagnóstico está basado en el aspecto histopatológico y el tratamiento consiste en la remoción quirúrgica. Se presenta el caso de una paciente mujer de 58 años edad con un adenoma pleomórfico de aproximadamente $3 \mathrm{~cm}$ localizado en el paladar, que fue tratado por escisión quirúrgica.

Palabras clave: adenoma pleomórfico; tumor de glándulas salivales; glándulas salivales menores.

\section{Abstract}

Pleomorfhic adenoma is the most common salivary gland neoplasm in both the major and minor salivary glands. It presents like a solid tumor of slow growth and asymptomatic. On minor salivary glands, the site more often affected is the hard palate, followed by the upper lip and buccal mucosa. The diagnosis is base on the histopathology features and the treatment consists at surgical excision. Here, we report a clinical case of a female 58 years old patient with a pleomorfhic adenoma, of $3 \mathrm{~cm}$ in dimension in the upper palate, that was treated by surgical treatment.

Key words: Pleomorfhic Adenoma; tumors of salivary glands; accessory glands

\section{Daniel A. Carretero Ancelmo ${ }^{1}$ Humberto Silva Rubio ${ }^{2}$}

\author{
${ }^{1}$ Cirujano Dentista Egresado de la Facultad de \\ Odontología de la UNMSM \\ 2 Jefe del Dpto. de Cirugía Oral y Maxilofacial \\ del Hospital Central Luis N. Saenz PNP.
}

Correspondencia:

Daniel Carretero Ancelmo

Calle José Sabogal No 183. Urb. Lucyana. Carabayllo

Teléfono: 0992-405272

Correo electrónico: himavidi2211@hotmail.com

Fecha de recepción:14-04-12

Fecha de aceptación: 06-09-12

\section{Introducción}

Existen tres glándulas salivales principales (parótida, submandibular y sublingual), así como innumerables glándulas salivales menores distribuidos por toda la mucosa de la cavidad bucal. Todas las glándulas, especialmente las principales o mayores, pueden ser objeto de inflamación o del desarrollo de neoplasias. ${ }^{1}$ Dada su morfología normal poco compleja de las glándulas salivales dan lugar a una sorprendente variedad de tumores benignos y malignos. ${ }^{2,3}$

El adenoma pleomórfico es el más frecuente de los tumores benignos de la glándula salival, compuesto fundamentalmente por una proliferación de células mioepiteliales y por un amplio espectro de componentes del tejido epitelial y mesenquimal, y rodeado por una nítida cápsula fibrosa. Pleomorfo se refiere a la amplia variabilidad de la diferenciación parenquimatosa y estromal por las células tumorales, no confundir con pleomorfismo nuclear exhibido por neoplasia maligna. Representa el $60 \%$ de los tumores de la glándula parótida, el $50 \%$ de los tumores de la glándula mandibular y solo el $25 \%$ de las neoplasias de la glándula sublingual.,
El $50 \%$ de los tumores de las glándulas menores orales son tumores mixtos, de los cuales un $55 \%$ se localizan en el paladar, un $25 \%$ en el labio superior, un $10 \%$ en la mucosa labial y un $10 \%$ en el resto de zonas orales y oro faríngeas. ${ }^{6}$

Clínicamente el adenoma pleomórfico de las glándulas menores se presenta como una masilla de crecimiento lento, firme, bien delimitada e indolora. ${ }^{1}$ Es blando o ligeramente firme a la palpación y libremente desplazable en las glándulas salivales mayores. Los tumores del lóbulo profundo no siempre se manifiestan por una masa facial. En ocasiones, tumores existentes desde hace muchos años se hacen lobulados o multilobulados. En las glándulas menores orales la presentación más frecuente es una tumefacción blanda y ligeramente indurada en el paladar blando o duro, con ulceración y telangiectasias de la mucosa que la recubre. ${ }^{5}$ Aparece en pacientes de cualquier edad; el $60 \%$ de los casos se dan entre la $3^{a}$ y $5^{a}$ década de vida, la edad media es 40 ańos y menos del $10 \%$ se producen en niños. $\mathrm{El}$ cociente $\mathrm{H}: \mathrm{M}$ es superior a 2:1. La resonancia magnética nuclear (RMN) es una técnica diagnóstica fiable para determinar la extensión de la enferme- dad, especialmente en las glándulas salivales mayores. ${ }^{4,6,7}$

El adenoma pleomórfico es mejor tratado por escisión quirúrgica. En las glándulas menores la lesión suele ser fácilmente enucleada mediante una escisión local. La transformación de este tumor es raramente relatada y cuando esto ocurre, el tumor pasa a ser denominado carcinoma ex adenoma pleomórfico. ${ }^{6,8}$ Este cáncer se encuentra dentro de las neoplasias más agresivas de las glándulas salivales, siendo responsables de un 30 a un $50 \%$ de mortalidad a los 5 años. ${ }^{\text {? }}$

La tasa de transformación maligna es incierta, pero diversos investigadores han estimado que es del 3 al $15 \%$. Livolsi y Perzin estudiaron 47 de dichos casos, presentes tanto en las glándulas mayores como en las menores.

La probabilidad de que un tumor de una glándula salival sea maligno es inversamente proporcional al tamaño de la glándula. Generalmente, cuando se diagnostican por primera vez, tanto las lesiones benignas como las malignas oscilan entre los 4 a $6 \mathrm{~cm}$. de diámetro, y son móviles con la palpación excepto en caso de tumores malignos desaten- 
didos. La mayor parte de los adenomas pleomórficos se presentan en forma de masas básicamente redondeadas, bien delimitadas, que rara vez superan los $6 \mathrm{~cm}$ de diámetro mayor. Aunque se sabe que los tumores benignos están presentes varios meses o ańos antes de que el paciente solicite evaluación clínica. Los canceres parecen hacerlo con mayor prontitud, posiblemente debido a su crecimiento más rápido. Se requiere siempre una evaluación morfológica para diferenciar lesiones benignas de las malignas. ${ }^{1,5}$

El objetivo del presente artículo es presentar un caso y revisar las características clínicas, criterios diagnósticos y tratamiento de la patología.

\section{Reporte de caso}

\section{a) Anamnesis}

Paciente femenino de 57 años de edad natural de Cañete, raza negra, acude a la consulta privada por presentar lesión en la encía a nivel postero superior izquierdo maxilar y exodoncia de pza. 2.8 .

La paciente refiere que desde hace cinco ańos presenta aumento de volumen de inicio repentino, sin dolor, lento y progresivo que la dificulta ingerir sus alimentos. Presenta antecedente de fiebre tifoidea, histerectomía y recibió transfusión sanguínea. En los antecedentes

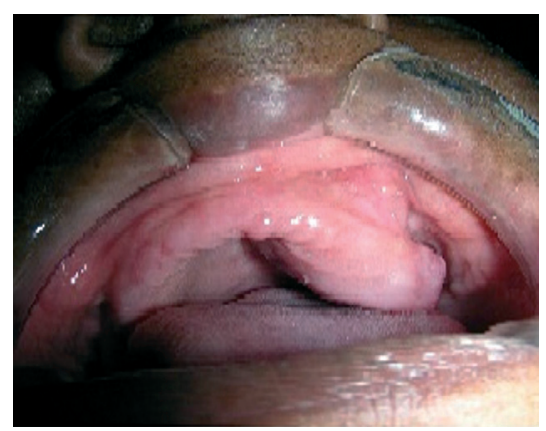

Fig 1. Vista intraoral de la tumoración en el paladar.

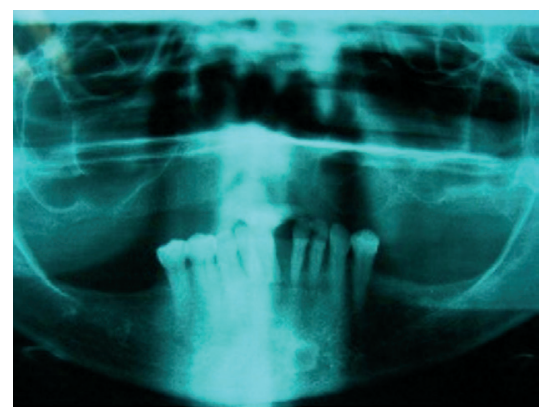

Fig 4. Alteración del seno maxilar. familiares informó que el tío presentó cáncer a los 72 años, el padre cáncer al estómago a los 60 ańos y la madre infarto al miocardio y epilepsia. Los demás familiares aparentemente sanos.

\section{b) Examen clínico}

El paciente se presento con funciones vitales dentro de los parámetros normales, hidratada, lucida, sin pérdida de peso y deambula sin dificultad.

$\mathrm{Al}$ examen extraoral: lunar de $5 \mathrm{~mm}$ de diámetro en la comisura labial del lado derecho sector superior, colapso oclusal con queilitis angular sin adenopatías a la palpación submaxilar ni cervical.

$\mathrm{Al}$ examen intraoral presencia de tumor que ocupa el sector 22 - 27 en paladar duro que se extiende a surco vestibular de $3 \mathrm{~cm}$ de diámetro consistencia blanda, sésil, ulcerado e indoloro a la exploración (parestesia). No movilidad de pieza dentaria del lado comprometido. (Fig. 1)

\section{c) Diagnóstico}

Radiográficamente se observa destrucción ósea, y la lesión presentaba bordes definidos circulares radiolúcidos, una zona de $25 \times 15 \mathrm{~mm}^{2}$ de área, imagen lobulada y altera el seno maxilar izquierdo a nivel de las pzas. 23- 26. (Fig.3 - 5)

Se realizó la biopsia por incisión del tumor y el resultado del examen microscópico presentó proliferación de células epiteliales dispuesta en mantos y

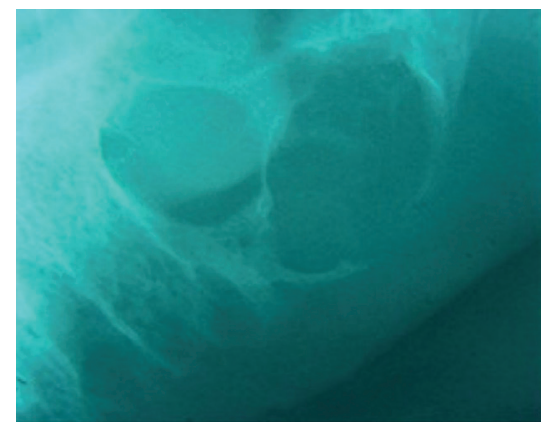

Fig 2. Bordes definidos circulares a la imagen radiográfica.

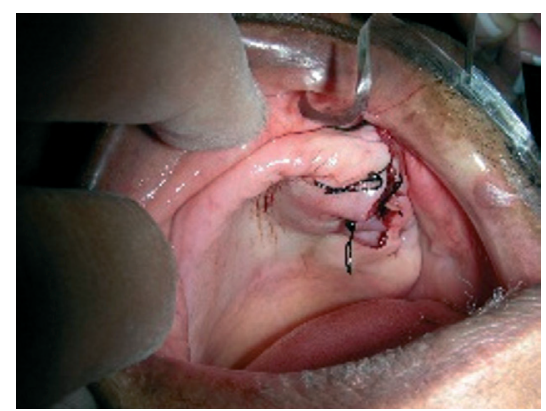

Fig 5. Sutura de la herida operatoria. estructuras ductales dentro de una matriz mixocondroide y con áreas de hialinización, en algunas zonas se distingue metaplasma escamosa con formación de estructuras queratinizadas. Los márgenes de sección quirúrgica en profundidad estaban comprometidos. Por lo cual se llega al diagnóstico de adenoma pleomórfico con metaplasia escamosa.

Los exámenes hematológico y bioquímico estuvieron dentro de parámetros normales.

\section{d) Plan de tratamiento, evolución y resultados}

El paciente fue referido al Hospital Nacional Central Luis N. Sáenz PNP al servicio de Cirugía Oral y Maxilofacial para su tratamiento el cual consistió en la extirpación quirúrgica de la lesión con evolución favorable tras cuatro meses de post operatorio. (Fig. 5 y 6 )

\section{Discusión}

El adenoma pleomórfico puede ocurrir a cualquier edad, pero es más común entre los 30 y 50 años de edad. Existe predilección por el sexo femenino $2: 1 .^{6,7}$

La paciente en cuestión es del sexo femenino, confirmando la proporción estadística relatada en la literatura. Sin embargo presenta edad superior a la presentada en la literatura consultada.

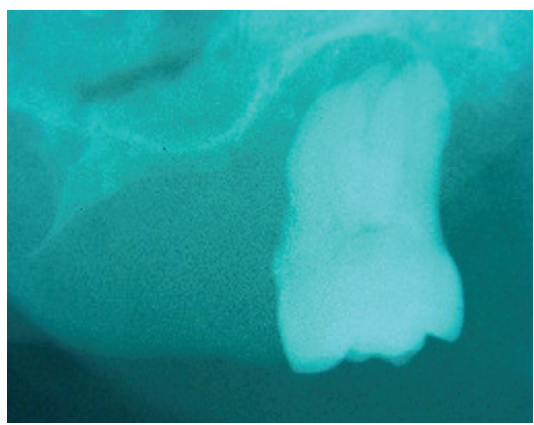

Fig 3. Imagen de la pieza implicada.

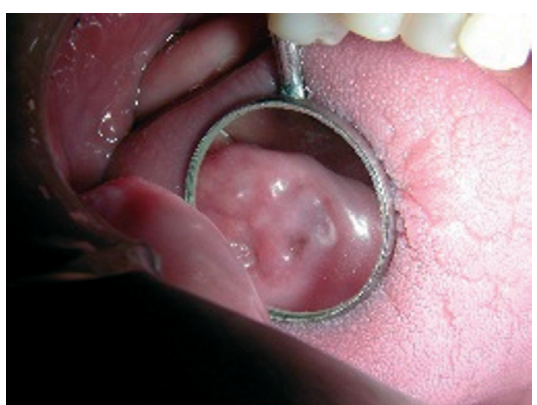

Fig 6. Control a los cuatro meses. 
Neville et al. citan que este tumor representa el $38 \%$ a los $43 \%$ de los tumores de las glándulas salivales menores, ocurriendo con mayor frecuencia en el paladar, seguido del labio superior y la mucosa bucal. ${ }^{6}$ La ubicación del tumor reportado coincide con la ubicación más común, según lo descrito en la literatura.

La historia relatada por la paciente de lesión nodular inicial pequeña, sin movilidad, indolora que empezó a aumentar de tamaño dirige el diagnóstico hacia adenoma pleomórfico. Rara vez la piel se ulcera, el dolor no es un síntoma común en el adenoma pleomórfico. ${ }^{3}$ Clínicamente varias lesiones suelen hacer diagnóstico diferencial con adenoma pleomórfico, como lipoma, quistes, neuroma y otros tumores de glándulas salivales. ${ }^{9}$ Tal es el caso presentado ya que la paciente refería las mismas características clínicas, incluso la ulceración reportado en la literatura.

El adenoma pleomórfico de las glándulas salivales accesorias intrabucales raramente obtiene un diámetro superior a 1 o $2 \mathrm{~cm}$. Por el hecho este tumor causa dificultades de masticación, habla y respiración, él es descubierto y tratado mas precozmente que los tumores de las glándulas principales. ${ }^{10}$ En este trabajo la lesión es de $3 \mathrm{~cm}$ y por su localización ocasionaba dificultad para ingerir sus alimentos a la paciente.

Ante la sospecha clínica de adenoma pleomórfico debe ser confirmada por examen histopatológico. ${ }^{11}$ En este caso podemos observar al examen microscópico proliferación de células epiteliales dispuesta en mantos y estructuras ductales dentro de una matriz mixocondroide y con áreas de hialinización, en algunas zonas se distingue metaplasma escamosa con formación de estructuras queratinizadas que permite diferenciarlo. Este patrón es similar a lo descrito en la literatura, el cual menciona que histológicamente hay presencia de capsula fibrosa, patrones de diferenciación ductal y mioepitelial. ${ }^{4}$ Están compuestos por elementos epiteliales dispersos en una matriz de tejido hialino, mixoide, condroide (cartilaginoide incluso óseo). ${ }^{5}$ Los tumores de glándulas salivales son un desafío especial para el patólogo. Su causa está en parte por la complejidad de la clasificación y por su rareza. El adenoma pleomórfico tiene la descripción histopatológica más completa de los tumores de glándulas salivales. Sin embargo la heterogeneidad de las características puede causar confusión y dificultad, principalmente en biopsias menores. ${ }^{12}$ Áreas del adenoma pleomórfico suelen ser semejantes o idénticas a una variedad de otros tipos de tumores incluyendo el adenocarcinoma polimorfo de bajo grado, el carcinoma adenoide quístico y el carcinoma epitelial - mioepitelial. El patólogo debe considerar el sitio y la historia clínica, pero en algunos casos la diversidad de las características morfológicas de la lesión suelen ser concluyentes solo cuando la lesión fue removida completamente y examinada por completo. ${ }^{13}$ El adenoma pleomórfico cuando es removido a través de procesos quirúrgicos apropiados, tiene un pronóstico excelente, existiendo un índice de recuperación de la patología alrededor del $95 \%$. En este caso, el adenoma pleomórfico fue removido quirúrgicamente con anestesia local, demostrando así que esta condición tiene un pronóstico favorable desde que se instaura el tratamiento adecuado con un examen clínico riguroso. ${ }^{6}$

En general, las lesiones del paladar duro deben quitarse junto con la mucosa que lo recubre, en tanto que las que se encuentran en mucosa de revestimiento, como en los labios, paladar blando y mucosa bucal muchas veces son tratados con éxito mediante enucleación o la escisión extracapsular. Como los tumores son radio resistentes, tiene poco beneficio la radioterapia razón por la que está contraindicada.,

La sospecha del adenoma pleomórfico ha sido confirmada a través del examen clínico radiográfico e histopatológico, siendo la paciente sometida al tratamiento quirúrgico de acuerdo con lo consultado en la literatura. Pasado 4 meses después de la cirugía, no existen señales de recidiva indicando éxito en el tratamiento.

\section{Conclusiones}

El odontólogo debe conocer, identificar y correlacionar los signos clínicos para poder diferenciar una neoplasia benigna de una maligna. Además de proceder a tomar una biopsia de rutina y hacer un examen histopatológico de la lesión para descartarla.

\section{Referencias Bibliográficas}

1. Shafer W., Hine M., Levy, B., Tratado de Patología Bucal, $4^{\circ}$ Edición, Rio de Janeiro 1987; 3: 213 - 218

2. Ellis G, Auclair P. tumors of the salivary glands. Atlas of tumor pathology, third series, fascicle 17 . Washington, DC, FORMED forcer. Institute of pathology, 1996.

3. Nagler R, Lanfer D. tumors of the major and minor sallivary glands: review of 25 years of experience. Anticancer res 1997; 17:701.

4. Sapp P. Patología oral y maxilofacial contemporánea. Ediciones Harcourt ESPANAA. Madrid 1999: 338 - 41.

5. Robbins y Cotran. Patología Estructural y Funcional. $7^{\mathrm{a}}$ Edición. Madrid. Editorial Elsevier S.A.; 2007: $460-62$.

6. Neville B.,Damm D., Allen C.,Bouquot J., Patología Oral e Maxilofacial. 2a Ed. Rio de Janeiro: Guanabara Koogan, 2004.

7. Regezi J, Sciubba J. Texto de Patología Bucal. 1a Ed. País: Interamericana- McGraw-Hill; 1989: $137-$ 140.

8. Silva M.,Kosgodak.,Tilakaratne W., Murugadas P.: A case of giant pleomorfhic adenoma of parotid gland. Oral Oncology. (2004); 40: 43 - 5.

9. Speight P., Barret A.: Salivary glands and saliva. Oral Dis. (2002) 8: 229 -240 .

10. Louro R.S., Passeado D., Andrade M, Medeiro P.J, Kobler.: Adenoma Pleomórfico em palato duro: relato de un caso.RBO,(2002); 59: $25-$ 27.

11. Chau M.,Radden B.: A clinical pathological study of 53 intra - oral pleomorfhic adenomas. Int J Oral Max Surg. (1989); 18: 158 - 62.

12. Souza S.O.M.,Araujo V.C.:Estudo morfologico e imuno - histoquimico do adenoma pleomórfico de glándula salivar menor. RPG. (1994); 1: $22-26$.

13. Yib W., Kratochvil F., Stewart J.: Intraoral minor salivary glands neoplasms: reviews of 213 cases. J Oral Maxillofac Surg. (2005); 63: 805 10. 